\title{
Encuesta para valorar la percepción de los estudiantes de medicina veterinaria sobre las estrategias didácticas recibidas en su formación profesional en una universidad particular de Lima - Perú.
}

Survey to assess the perception of veterinary medicine students on the didactic strategies received in their professional training at a private university in Lima - Peru.

\section{Rodrigo Rondón Herz ${ }^{1}$, Carlos-Augusto Echaiz Rodas ${ }^{1}$}

\section{RESUMEN}

El objetivo del estudio fue construir y validar una encuesta piloto, para medir la percepción de los estudiantes de medicina veterinaria acerca de las estrategias didácticas recibidas en su formación profesional, en la Universidad Peruana de Ciencias Aplicadas de Lima-Perú. El tipo de estudio fue observacional, descriptivo, y correlacional. El instrumento evaluó las dimensiones de la variable estrategias didácticas (percepción docente, cognitivos, procedimental y actitudinal). Asimismo, este cuestionario recibió la validación de contenido por medio del juicio de tres expertos, y se aplicó a 30 estudiantes, para verificar la redacción de los ítems. Luego se aplicó a una muestra de 137 estudiantes. La confiabilidad interna se definió a través de la determinación del Coeficiente de Alfa de Cronbach (CAC) Global $(0,87)$ y para cada ítem. Además, se determinó la pertinencia de la aplicación del Análisis Factorial Exploratorio (AFE), por medio de la determinación del Indice kaiser Meyer Olkin $(\mathrm{KMO}=0,65)$. Se realizó el Análisis de Componentes Principal y la gráfica de sedimentación, para determinar el número de factores a extraer. El AFE determinó que hay preguntas específicas que están correlacionadas tanto para cada indicador de cada una de las dimensiones de la variable estrategias didácticas como para cada uno de los cinco factores extraídos. Además, el CAC determinado por factor mostró un nivel de consistencia interna entre aceptable $(0,66)$ y elevado $(0,88)$, y la organización de la estructura factorial con rotación varimax, permitió identificar los indicadores por dimensión que pueden ser medidos para cada uno de los factores. De esta forma, las dimensiones que se miden por factor son: 1) Percepción docente y procedimental; 2) Percepción docente, cognitivos y actitudinal; 3) Percepción docente y actitudinal; y 4) Cognitivo y procedimental.

PALABRAS CLAVE: Estrategias didácticas, habilidades clínicas, medicina veterinaria, educación. 


\section{SUMMARY}

The objective of the study was to construct and validate a pilot survey to measure the perception of veterinary medicine students about the didactic strategies received in their professional training at the Peruvian University of Applied Sciences in Lima-Peru. The type of study was observational, descriptive and correlation's. The instrument evaluated the dimensions of the didactic strategies variable (teaching perception, cognitive, procedural and attitudinal). Likewise, this questionnaire received content validation through the judgment of three experts, and was applied to 30 students to verify the wording of the items. It was then applied to a sample of 137 students. The internal reliability was defined through the determination of the Cronbach Alpha Coefficient (CAC) Overall (0.87) and for each item. In addition, the relevance of the application of the Exploratory Factor Analysis (EFA) was determined through the determination of the Kaiser Meyer Olkin Index $(\mathrm{KMO}=0.65)$. Principal Component Analysis and the sedimentation plot were performed to determine the number of factors to be extracted. The PCA determined that there are specific questions that are correlated both for each indicator of each of the dimensions of the variable didactic strategies and for each of the five factors extracted. In addition, the CAC determined by factor showed an acceptable (0.66) to high (0.88) level of internal consistency, and the organization of the factorial structure with varimax rotation made it possible to identify the indicators per dimension that can be measured for each of the factors. Thus, the dimensions measured by factor are: 1) Teacher and procedure perception; 2) Teacher perception, cognitive and attitudinal; 3) Teacher and attitude perception; and 4) Cognitive and procedural.

KEY WORDS: Didactic strategies, clinical skills, veterinary medicine, education.

\section{INTRODUCCIÓN}

La medicina veterinaria es una ciencia, que tiene como misión dar respuesta a problemas en las áreas de la salud y producción animal, salud pública desde el campo de la veterinaria, inocuidad de alimentos destinados para el consumo humano, biodiversidad de las especies, manejo de ecosistemas para contribuir al desarrollo sostenible de la sociedad y el ambiente, entre otras responsabilidades (Izaga, 2018). Dentro de un contexto más específico, esta ciencia médica engloba gran cantidad de conocimientos para fortalecer la capacitación integral del médico veterinario, tanto en los procedimientos para lograr un diagnóstico clínico como en el uso eficiente de los medicamentos para el control de las diferentes patologías que se deben atender, y en las orientaciones adecuadas sobre prevención y cuidado que les debe brindar a los propietarios de los animales (San Martín, 2003; Allen et al., 2007).

En este sentido, la calidad del profesional en esta área depende de una adecuada formación profesional. Es así como uno de los grandes retos que ha confrontado la educación superior veterinaria, y que aún continua vigente, es integrar los conocimientos teóricos a la práctica para la intervención y resolución de problemáticas inherentes a la realidad (Taylor, 2012). Además, el aporte a la la solución de este reto debe darse a través de la implementación de una estructura curricular que se enfoque en fortalecer las competencias del profesional que egresa de los centros de estudios (Barreda, 2009).

Uno de los cambios que se plantean a nivel educativo es la construcción de un currículo en base a competencias. Villa y Poblete (2004) definen a la competencia como "un buen desempeño en contextos complejos y auténticos. Se basa en la integración y activación de conocimientos, habilidades y destrezas, actitudes y valores" y López (2016) describe que la competencia tiene tres dimensiones: conocimiento conceptual (son las habilidades cognitivas, referidas al saber conocer), conocimiento procedimental (son las capacidades, habilidades prácticas y destrezas, y están referidas al saber hacer) y conocimiento actitudinal (se refiere a las motivaciones, emociones y valores, y están entrelazadas con el saber ser). Asimismo, el "para qué" de la competencia tiene que ver con el ejercer y realizar tareas o actividades, dentro de un contexto o una dimensión social.

La necesidad de implementar el modelo educativo por competencia ha involucrado cambios en los métodos, técnicas y estrategias de aprendizajes que se vinculan con el proceso de enseñanza - aprendizajes, y las metodologías de evaluación (Mazur, 2009), modificando el modelo de enseñanza que se centra en el docente, es decir, que él tiene todos los conocimientos y el educando no (King, 1993), por uno que plantea el modelo de competencias bajo el enfoque constructivista, el cual propone un modelo 
centrado en la enseñanza, donde el educando tiene un papel activo, y este es considerado como un ser que ya tiene conocimiento (Cooper et al., 2015).

En este orden de ideas, y según Piaget (1964) citado por Kim et al. (2019, pág. 30) señala que: "Los constructivistas argumentan que el conocimiento se forma y se retiene cuando el individuo da sentido a la nueva información en el contexto de la información existente". De esta forma el uso de estrategias didácticas le permite al educando participar, procesar y aplicar activamente lo aprendido (King, 1993), y en consecuencia él adquiere las nuevas herramientas que lo aproximan al proceso de transición hacia el mundo profesional (Kim et al., 2019).

En este sentido, la preparación del médico veterinario depende en gran parte de la calidad del proceso enseñanza - aprendizaje que recibe en su centro de formación académica y en este proceso, el docente juega un rol importante. Por ello, al docente se le exige un buen nivel de preparación académica, estar actualizado en cuanto al manejo de la información y tener disposición para formar al educando. Asimismo, la organización del proceso de enseñanza se verá favorecido por el enfoque didáctico que se use. Por tanto, contar con instrumentos de medición, para evaluar el proceso de enseñanza - aprendizaje, contribuirá con la identificación de las debilidades y fortaleza del mismo, y aportará elementos teóricos para lograr la certificación del proceso de formación académica del educando. Por ello, el objetivo del estudio fue construir y validar una encuesta piloto para medir la percepción de los estudiantes de medicina veterinaria sobre las estrategias didácticas que han recibido durante los diferentes ciclos académicos cursados en la Universidad Peruana de Ciencias Aplicadas (UPC).

\section{MATERIAL Y METODOS}

La investigación correspondió a un estudio no experimental, observacional de nivel descriptivo, correlacional con un enfoque cuantitativo (Álvarez y Delgado, 2015). La población objetivo estuvo conformada por 167 estudiantes del segundo al octavo ciclo de la malla curricular de la carrera de Medicina Veterinaria de la UPC, matriculados hasta el año académico 2021.

Los criterios de inclusión en el estudio fueron los siguientes: que fueran estudiantes de la carrera de medicina veterinaria de la universidad seleccionada, tener una edad igual o mayor a 18 años y encontrase cursando entre el segundo y octavo ciclo de la malla curricular de su formación profesional. Los criterios de exclusión fueron no estar de acuerdo en participar en el estudio y consecuentemente no firmar el consentimiento correspondiente.

En cuanto al diseño del instrumento, inicialmente fue construido con 17 preguntas, auto aplicado, valorado en una escala tipo Likert, que van desde completamente de acuerdo (5), de acuerdo (4), ni de acuerdo ni en desacuerdo (3), en desacuerdo, hasta complemente en desacuerdo (1). Se construyó bajo la base teórica de los modelos de competencia constructivista. En este sentido, este modelo se inscribe, según Andrade (2020), "dentro de los modelos de aprendizaje centrados en el aprendizaje, porque pone énfasis en metodologías activas, en donde el estudiante se compromete en el desarrollo de sus procesos cognoscitivos y asume una posición crítica y reflexiva ante su propio aprendizaje". Asimismo, junto a este enfoque teórico y al de metodología activa, se evalúo las estrategias didácticas y su relación con las habilidades clínicas.

Esta investigación parte de reconocer a la práctica clínica como una acción donde los estudiante o profesionales de la salud aplican la enseñanza teórica que han aprendidos, para resolver una situación concreta que se relaciona con el cuidado del paciente (García-Carpintero et al., 2019). Asimismo, la estrategia didáctica se define, según Tebar (2003) citado por Flores et al. (2017), como "procedimientos que el agente de enseñanza utiliza en forma reflexiva y flexible para promover el logro de aprendizajes significativos en los estudiantes". Dentro de este enfoque, el educador es el responsable de diseñar, planificar y organizar todo el escenario necesario para que se dé el aprendizaje y se puedan alcanzar las competencias que se han definido como metas. Además, otros de las características que deben estar presentes en el educando, tienen que ver con su responsabilidad de Identificar, interpretar, argumentar, resolver problemas y poner en práctica lo aprendido, y actualizar lo que ya sabe (López, 2016). Por último, se consideró a las rubricas como un instrumento de evaluación desde la mirada del docente y el educando, para valorar de forma objetiva y concreta las competencias alcanzadas a partir del proceso de enseñanza aprendizaje desarrollado (Bertone, 2019).

Partiendo del contexto teórico indicado en los párrafos anteriores, se definió operacionalmente 
la variable estrategia didáctica. Así, la encuesta se conformó por las siguientes dimensiones, indicadores y preguntas (•):

\section{DIMENSIÓN: PRECEPCIÓN DOCENTE}

- Indicador 1: Contenido del curso

- La información previa al examen fue completa y satisfactoria $(\mathrm{P} 2)$

- Indicador 2: Estrategias de enseñanza

- La información brindada por el docente fue en lenguaje claro (P3)

- La enseñanza del curso colmo mis expectativas (P4)

- $\quad$ El tiempo de la clase se aprovecha al máximo (P7)

- Se cumple con el horario y se facilita el autoaprendizaje (P8)

- Indicador 3: Estrategias de aprendizaje

- El docente trata de manera correcta a los alumnos, se muestra accesible a escuchar sugerencias y consultas y las atiende de manera adecuada (P6)

- $\quad$ El uso de herramientas tecnológicas ayuda a mi aprendizaje del curso (P9)

\section{DIMENSIÓN: COGNITIVAS}

- Indicador1: Evaluación con rúbricas

- La evaluación con rúbricas es adecuada para medir tus aprendizajes cognitivos (P10)

- Indicador 2: Conocimientos adquiridos

- Los conocimientos que estás adquiriendo te permiten tomar decisiones adecuadas para la ejecución de tus actividades de tu práctica clínica actual (P12)

- Indicador 3: Formación para el campo laboral profesional

- Los conocimientos que estás adquiriendo te permitirán tomar decisiones adecuadas cuando seas profesional (P11)

\section{DIMENSIÓN: PROCEDIMENTAL}

- Indicador 1: Desarrollo de competencias en cursos

- El diseño curricular por competencias te permite el adecuado desarrollo de destrezas y habilidades clínicas (P13)

- Indicador 2: Evaluación con rúbricas

- La evaluación con rúbricas es adecuada para medir tu desarrollo de destrezas y habilidades clínicas (P14)

- Indicador 3: Desarrollo de destrezas

- Las destrezas procedimentales que estás desarrollando te permiten desempeñarte adecuadamente en la ejecución de tus actividades de práctica clínica actual (P15)

- Indicador 4: Formación para el campo laboral profesional

- Las destrezas procedimentales que estás desarrollando te permitirán desempeñarte adecuadamente en la ejecución de actividades clínicas cuando seas profesional (P16)

\section{DIMENSIÓN: ACTITUDINAL}

- Indicador 1: Diseño curricular por competencias

- $\quad$ El diseño de tus cursos promueve el adecuado desarrollo de actitudes y valores para el ejercicio profesional (P17)

- Indicador 2: Desarrollo de competencias en cursos

- Los criterios y método de evaluación de la asignatura han sido claramente comunicados (P1)

- Indicador 3: Evaluación con rúbricas

- Las preguntas que realizo en clase fueron respondidas de manera satisfactoria (P5)

Una vez construido la encuesta, se realizó la validación de contenido por medio de tres jueces (expertos en el área de estudio), para después, aplicar el instrumento a una muestra piloto de 30 estudiantes, con el objeto de hacer ajustes o modificaciones sugeridas por los jueces y estudiantes.

La organización de los datos obtenidos de la aplicación de la encuesta se realizó en el programa Microsoft Excel y el análisis descriptivo e inferencial de los datos se realizó a través del software Stata (versión 15). En cuanto al paso a paso, se siguió la metodología definida por Lemos y Londoño (2006):

1. Se evaluó la consistencia interna del instrumento a través de la determinación del Coeficiente Alfa de Cronbach, el punto de corte fue valor superior a 0.6 .

2. El análisis factorial exploratorio (AFE) se llevó a cabo con el método de componentes principales (ACP) con rotación ortogonal varimax. Asimismo, se realizó la gráfica de sedimentación para corroborar el número de factores que se habían definido en el ACP, y que debían ser extraídos, al correr el AFE.

3. Se calculó el índice de Káiser - Meyer - Olkin (KMO), y como criterio se definió que el índice debería ser mayor que 0.6 para justificar la realización del AFE.

4. Para la elección de los factores y los ítems que lo conforman, se consideraron los siguientes criterios: - Los factores debían tener un valor propio 
mayor que 1 ó una varianza acumulada superior a $70 \%$.

- $\quad$ La pregunta debía tener una saturación (carga factorial) igual o superior a 0.50 .

- La pregunta debía estar incluida o relacionada con un solo factor; aquel en el que presentara un mayor nivel de saturación, y preguntas con cargas similares en distintos factores fueron excluidas.

- Debía poseer una congruencia conceptual entre todas las preguntas que se incluyeran en un factor.

- Un factor debía estar conformado por tres o más preguntas, a excepción de aquellos factores en el que dos preguntas estuvieran claramente sustentadas por la teoría o por el coeficiente de consistencia interna. Un factor debía poseer una confiabilidad o un valor de Coeficiente de Alfa de Cronbach superior a 0,5 .

5. Se les aplicó la encuesta a los 137 estudiantes restante, para medir la percepción de los estudiantes de la carrera de medicina veterinaria sobre las estrategias didácticas que han recibido en los diferentes ciclos académicos que están cursando.

El protocolo fue presentado al Sub Comité de Ética de la UPC para su respectiva evaluación. Asimismo, a los participantes del estudio se le entregó y explicó el contenido del consentimiento informado antes de iniciar el proceso de aplicación de la la encuesta. También, fueron respetadas las reglas internas y externas sobre la elaboración y desarrollos de trabajos de investigación.

\section{RESULTADOS}

La población de estudio comprendió a los estudiantes matriculados entre los ciclos 2 do y $8 \mathrm{vo}$ de la carrera de medicina veterinaria de la UPC. Participaron 167 estudiantes de los cuales 128 (76,6\%) fueron mujeres y $39(23,4 \%)$ hombres, con edades entre 18 y 23 años. La distribución de los estudiantes según sexo y ciclo de estudios se presenta en la tabla 1.

Una vez que se obtuvo la validez de contenido por parte de los jueces y se hicieron los ajustes sobre la primera aplicación del instrumento a los 30 estudiantes de la población de estudio, se procedió con la segunda aplicación de la encuesta a los 137 estudiantes restantes de la población total. El siguiente paso fue obtener los resultados del análisis del Coeficiente Alfa de Cronbach, los cuales muestran valores superiores a 0.8 para todos los ítems y un alfa global de 0,87 (tabla 2). Estos resultados indican que los reactivos del instrumento muestran una buena consistencia interna de forma individual y global.

Asimismo, los Análisis de Componentes Principales (tabla 3) y la Gráfica de Sedimentación (figura 1) permitieron establecer como criterio, la selección de 5 componentes o factores a extraer. También, se determinó el valor del índice de Káiser - Meyer - Olkin (KMO), el cual fue igual a 0.65, confirmando que los datos se ajustaban y se podía realizar el análisis factorial exploratorio (AFE).

Al realizar los AFE con los cinco componentes extraídos y la rotación varimax para el método de rotación ortogonal, quien minimiza el número de

Tabla 1. Descripción de la población de los estudiantes seleccionados de la carrera de Medicina Veterinaria de la Universidad Peruana de Ciencias Aplicadas en el 2021.

\begin{tabular}{|c|c|c|c|c|c|c|}
\hline \multirow{3}{*}{$\begin{array}{c}\text { Ciclos } \\
\text { académicos }\end{array}$} & \multicolumn{4}{|c|}{ Sexo Biológico } & \multirow{2}{*}{\multicolumn{2}{|c|}{ Total }} \\
\hline & \multicolumn{2}{|c|}{ Mujeres } & \multicolumn{2}{|c|}{ Hombres } & & \\
\hline & $\mathrm{n}$ & $\%$ & $\mathrm{n}$ & $\%$ & n & $\%$ \\
\hline $2 \mathrm{do}$ & 55 & 32,9 & 25 & 15,0 & 80 & 47,9 \\
\hline 3ero & 21 & 12,6 & 6 & 3,6 & 27 & 16,2 \\
\hline 4to & 17 & 10,2 & 3 & 1,8 & 20 & 12,0 \\
\hline 5 to & 15 & 9,0 & 2 & 1,2 & 17 & 10,2 \\
\hline 6to & 12 & 7,2 & 2 & 1,2 & 14 & 8,4 \\
\hline $7 \mathrm{mo}$ & 6 & 3,6 & 1 & 0,6 & 7 & 4,2 \\
\hline $8 \mathrm{vo}$ & 2 & 1,2 & 0 & 0 & 2 & 1,2 \\
\hline Total & 128 & 76,6 & 39 & 23,4 & 167 & 100 \\
\hline
\end{tabular}


Tabla 2. Resultados de la determinación del Alfa de Cronbach por ítem y global. Carrera de Medicina Veterinaria, Universidad Peruana de Ciencias Aplicadas 2021.

\begin{tabular}{cccccc}
\hline Preguntas & Observaciones & $\begin{array}{c}\text { Preguntas } \\
\text { - Test de } \\
\text { Correlación }\end{array}$ & $\begin{array}{c}\text { Preguntas } \\
\text { - Test de } \\
\text { Correlación }\end{array}$ & Covarianza & $\begin{array}{c}\text { Coeficiente } \\
\text { Alpha de } \\
\text { Cronbach }\end{array}$ \\
\hline P1 & 167 & 0,4663 & 0,3714 & 0,1198062 & 0,8777 \\
P2 & 167 & 0,7032 & 0,6582 & 0,1163234 & 0,8672 \\
P3 & 167 & 0,3457 & 0,2646 & 0,1252904 & 0,8800 \\
P4 & 167 & 0,7743 & 0,7279 & 0,1104947 & 0,8626 \\
P5 & 167 & 0,5053 & 0,4390 & 0,1212683 & 0,8742 \\
P6 & 167 & 0,3135 & 0,2529 & 0,1273778 & 0,8793 \\
P7 & 167 & 0,5684 & 0,4736 & 0,1146947 & 0,8740 \\
P8 & 167 & 0,5764 & 0,4940 & 0,1158803 & 0,8724 \\
P9 & 167 & 0,6780 & 0,5963 & 0,1094636 & 0,8681 \\
P10 & 167 & 0,5636 & 0,4858 & 0,1171819 & 0,8725 \\
P11 & 167 & 0,3974 & 0,3319 & 0,1248635 & 0,8774 \\
P12 & 167 & 0,6010 & 0,5488 & 0,1197295 & 0,8710 \\
P13 & 167 & 0,6771 & 0,5953 & 0,1094991 & 0,8682 \\
P14 & 167 & 0,5394 & 0,4642 & 0,1186960 & 0,8733 \\
P15 & 167 & 0,7461 & 0,6952 & 0,1114819 & 0,8640 \\
P16 & 167 & 0,7388 & 0,6888 & 0,1123551 & 0,8645 \\
P17 & 167 & 0,6872 & 0,6307 & 0,1142444 & 0,8669 \\
Test & & & & 0,1169795 & 0,8782 \\
\hline
\end{tabular}

Tabla 3. Resultados de análisis de componentes principales. Carrera de Medicina Veterinaria, Universidad Peruana de Ciencias Aplicadas 2021.

\begin{tabular}{ccccc}
\hline Componentes & $\begin{array}{c}\text { Valores } \\
\text { propios }\end{array}$ & Diferencias & Proporción & $\begin{array}{c}\text { Varianza } \\
\text { acumulada }\end{array}$ \\
\hline Comp1 & 6,10282 & 3,906810 & 0,3590 & 0,3590 \\
Comp2 & 2,19601 & 0,618628 & 0,1292 & 0,4882 \\
Comp3 & 1,577380 & 0,131907 & 0,0928 & 0,5810 \\
Comp4 & 1,445470 & 0,472878 & 0,0850 & 0,6660 \\
Comp5 & 0,972596 & 0,150460 & 0,0572 & 0,7232 \\
Comp6 & 0,822136 & 0,144827 & 0,0484 & 0,7716 \\
Comp7 & 0,677308 & 0,110216 & 0,0398 & 0,8114 \\
Comp8 & 0,567092 & 0,034663 & 0,0334 & 0,8448 \\
Comp9 & 0,532429 & 0,039435 & 0,0313 & 0,8761 \\
Comp10 & 0,492994 & 0,109825 & 0,0290 & 0,9051 \\
Comp11 & 0,383170 & 0,052924 & 0,0225 & 0,9276 \\
Comp12 & 0,330246 & 0,041594 & 0,0194 & 0,9470 \\
Comp13 & 0,288652 & 0,075578 & 0,0170 & 0,9640 \\
Comp14 & 0,213074 & 0,017173 & 0,0125 & 0,9766 \\
Comp15 & 0,195901 & 0,064077 & 0,0115 & 0,9881 \\
Comp16 & 0,131824 & 0,060928 & 0,0078 & 0,9958 \\
Comp17 & 0,070896 &. & 0,0042 & 1 \\
\hline
\end{tabular}




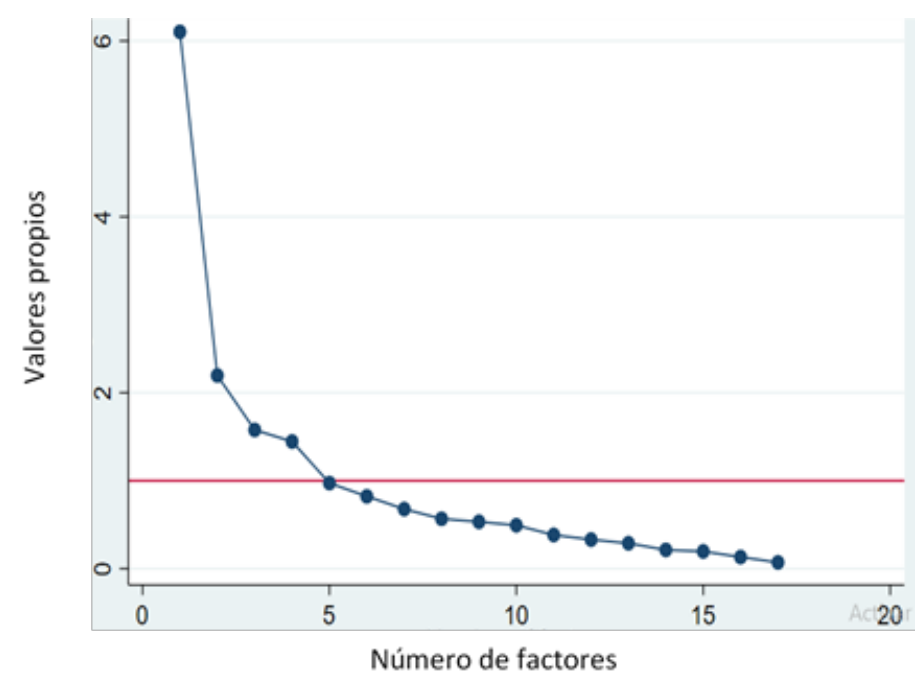

Gráfico 1. Gráfico de sedimentación de la encuesta piloto. Se identifican componentes con valores superiores a 1.

Tabla 4. Estructura factorial con rotación varimax de la encuesta que evalúa la percepción sobre las estrategias metodológicas, conformado por 17 ítems

\begin{tabular}{|c|c|c|c|c|c|}
\hline Preguntas & Factor 1 & Factor 2 & Factor 3 & Factor 4 & Factor 5 \\
\hline La enseñanza del curso colmo mis expectativas (P4). & 0,5682 & & & & \\
\hline El tiempo es el apropiado para el desempeño en la asignatura (P7). & 0,6881 & & & & \\
\hline Se cumple con los horarios establecidos (P8). & 0,8407 & & & & \\
\hline $\begin{array}{l}\text { El diseño curricular por competencias te permite el adecuado desarrollo de } \\
\text { destrezas y habilidades clínicas (P13). }\end{array}$ & 0,6954 & & & & \\
\hline
\end{tabular}

Las destrezas procedimentales que desarrollas te permiten desempeñarte adecuadamente en la ejecución de tus actividades de práctica clínica actual (P15).

Las destrezas procedimentales que desarrollas te permitirán desempeñarte adecuadamente en la ejecución de actividades clínicas cuando seas profesional (P16).

El uso de herramientas tecnológicas facilita el aprendizaje del curso (P9).

Los conocimientos adquiridos en clase son relevantes para la práctica clínica (P11).

Los conocimientos que estás adquiriendo te permitirán tomar decisiones adecuadas cuando seas profesional (P12).

El diseño de tus cursos promueve el adecuado desarrollo de actitudes y valores para el ejercicio profesional (P17).

Los criterios y método de evaluación han sido claramente comunicados (P1).

La información previa al examen fue completa y satisfactoria (P2).

La información brindada por el docente fue con un lenguaje claro (P3).

Las rúbricas utilizadas por los docentes evalúan correctamente las habilidades cognitivas (P10).

La evaluación con rúbricas es adecuada para medir tu desarrollo de destrezas y habilidades clínicas (P14). 
reactivos que tienen saturaciones altas en cada factor. Se encontró que para cada factor hay un número de preguntas que muestra una correlación con valores superior a 0.5 . Pero, hay dos casos en donde la correlación mostró valores de 0.4 , es decir, la pregunta 1, la cual está relacionada con el factor 3 y la pregunta 10 con el factor 4 (tabla 4). Además, se indican los factores y las dimensiones junto a sus indicadores que se pueden medir con el instrumento, así el:

- Factor1: Percepción docente [indicador: estrategia de enseñanza (2)] y procedimental [indicadores: desarrollo de competencias en cursos (1); desarrollo de destrezas (3); y formación para el campo laboral profesional (4)].
- Factor 2: Percepción docente [indicador: estrategias de aprendizaje (3)], cognitivos [indicadores: conocimientos adquiridos (2) y formación para el campo laboral profesional (3)], y actitudinal [indicador: diseño curricular por competencias (1)].

- Factor 3: Percepción docente [indicadores: contenido del curso (1) y estrategias de enseñanza (2)] y actitudinal [indicador: desarrollo de competencias en cursos (2)].

- Factor 4: Cognitivo [indicador: evaluación con rúbricas (1)]; y procedimental [Indicador: evaluación con rúbricas (2)].

- Factor 5: Actitudinal [indicador: evaluación con rúbricas (3)] y percepción docente [indicador: estrategias de aprendizaje (3)].

Tabla 5. Resultados de la determinación del Coeficiente de Alfa de Cronbach para cada uno de los componentes o factores extraídos del AFE.

\begin{tabular}{|c|c|c|c|}
\hline Factores & $\begin{array}{c}\text { Dimensiones de la variable estrategias } \\
\text { didácticas junto a } \\
\text { sus indicadores }\end{array}$ & Preguntas & $\begin{array}{c}\text { Coeficiente } \\
\text { Alfa de Cronbach } \\
\text { por dimensión } \\
\end{array}$ \\
\hline Factor 1 & Percepción Docente (2); Procedimental (1; 3; y 4). & $\begin{array}{l}\text { P4; P7; P8; P13; P15; } \\
\text { P16. }\end{array}$ & 0,8811 \\
\hline Factor 2 & $\begin{array}{c}\text { Percepción Docente (3); Cognitivos (2 y 3); y } \\
\text { Actitudinal (1). }\end{array}$ & P9; P11; P12; y P17. & 0,8269 \\
\hline Factor 3 & Percepción Docente (1 y 2); Actitudinal (2). & P1; P2; y P3. & 0,6849 \\
\hline Factor 4 & Cognitivo (1); Procedimental (2). & P10 y P14. & 0,6594 \\
\hline Factor 5 & Actitudinal (3); Percepción Docente (3). & P5 y P6. & 0,6731 \\
\hline
\end{tabular}

Tabla 6. Resultados de la determinación del Alfa de Cronbach para cada uno de los componentes o factores extraídos del AFE.

\begin{tabular}{ccccccccccc}
\hline \multirow{2}{*}{$\begin{array}{c}\text { Ciclos } \\
\text { académicos }\end{array}$} & \multicolumn{8}{c}{ Dimensión de la variable estrategias didácticas en cinco dimensiones o componentes } \\
& \multicolumn{2}{c}{ Factor 1* } & \multicolumn{3}{c}{ Factor 2* } & \multicolumn{2}{c}{ Factor 3* } & \multicolumn{2}{c}{ Factor 4* } & Factor 5* \\
& NA/ & DA/ & NA/ & DA/ & NA/ & DA/ & NA/ & DA/ & NA/ & DA/ \\
& ND & CDA & ND & CDA & ND & CDA & ND & CDA & ND & CDA \\
\hline 2do & 6 & 74 & 4 & 76 & 6 & 74 & 4 & 76 & 5 & 75 \\
3ero & 3 & 24 & 1 & 26 & 3 & 24 & 1 & 26 & 2 & 25 \\
4to & 2 & 18 & 3 & 17 & 2 & 18 & 3 & 17 & 1 & 19 \\
5to & 2 & 15 & 2 & 15 & 2 & 15 & 2 & 15 & 1 & 16 \\
6to & 1 & 13 & 2 & 12 & 1 & 13 & 2 & 12 & 1 & 13 \\
7 mo & 0 & 7 & 1 & 6 & 0 & 7 & 1 & 6 & 2 & 5 \\
8vo & 0 & 2 & 0 & 2 & 0 & 2 & 0 & 2 & 0 & 2 \\
Total & 40 & 127 & 30 & 137 & 14 & 153 & 13 & 154 & 12 & 155 \\
\hline
\end{tabular}

Nota: NA/ND: Ni de acuerdo ni en desacuerdo; DA/CDA: De acuerdo y Completamente de Acuerdo; Factor 1: Percepción Docente (2); Procedimental (1; 3; y 4); Factor 2: Percepción Docente (3); Cognitivos (2 y 3); y Actitudinal (1); Factor 3: Percepción Docente (1 y 2); Actitudinal (2); Factor 4: Cognitivo (1); Procedimental (2); y Factor 5: Actitudinal (3); Percepción Docente (3). 
Asimismo, el valor del CAC para cada factor mostró un nivel de fiabilidad que va desde aceptable a muy aceptable (tabla 5). También, se evidenció que hay factores que miden varias dimensiones y varios indicadores.

En cuanto a la aplicación de la encuesta en la segunda fase, se logró evidenciar que los estudiantes de la carrera de medicina veterinaria de la UPC de todos los ciclos académicos que participaron, tenian una buena percepción de las estrategias didácticas que se han desarrollado en clase (tabla 6).

\section{DISCUSIÓN}

La encuesta que ha sido elaborado en este proyecto permite medir la percepción de los estudiantes de la carrera de medicina veterinaria sobre las estrategias didácticas que han sido impartidas en sus diferentes ciclos académicos, y que a su vez están vinculadas al desarrollo de habilidades clínicas. En este sentido, el instrumento elaborado y validado en su fase inicial, permitió la definición de 5 factores, los cuales pueden ser considerado como 5 instrumentos, porque los resultados muestran que para cada factor hay un número de preguntas que miden una o varias dimensiones junto a sus indicadores, que conforman en su conjunto al constructo que define las estrategias didácticas. Asimismo, se evidenció que en cada factor se miden los siguientes indicadores:

- Factor 1: estrategia de enseñanza, desarrollo de competencias en cursos, desarrollo de destrezas, y formación para el campo laboral profesional.

- Factor 2: estrategias de aprendizaje, conocimientos adquiridos, formación para el campo laboral profesional, y diseño curricular por competencias.

- Factor 3: estrategias de enseñanza y desarrollo de competencias en cursos.

- Factor 4: evaluación con rúbricas.

- Factor 5: evaluación con rúbricas y estrategias de aprendizaje.

Uno de los aportes del instrumento que se diseñó, está vinculado con el hecho de que permite indagar sobre la valoración de la percepción del educando en cuanto a las estrategias de enseñanza y aprendizajes, las competencias o habilidades clínicas alcanzadas y el instrumento de evaluación implementado (rúbrica). Por tanto, el potencial de la encuesta está en la identificación de fortalezas y debilidades sobre el área de formación donde se aplique, con el fin de impulsar los cambios necesarios para mejorar la calidad del profesional en la carrera de medicina veterinaria que ha de egresar del centro de formación académica seleccionado para el estudio.

Este hallazgo de impulsar una reflexión, dentro de los centros de formación de educación superior, ya que como lo señala Hidalgo et al. (2017), muchos docentes del área de las Ciencias Médica, dominan ampliamente su carrera, pero adolecen de estrategias didácticas para facilitar el proceso de enseñanza - aprendizaje, por lo que se requiere un cambio en el modelo educativo, y junto a este el desarrollo de instrumentos que permitan medir o evaluar la calidad de la educación y el nivel de competencias que ha adquirido el educando durante su proceso de formación. En este sentido, Kim et al. (2019), señalan que la introducción de una agenda de trabajo como la desarrollada por Monash University (Universidad Australiana), es decir, "Mejor enseñanza, mejor aprendizaje" para ofrecer una experiencia de aprendizaje más centrada en el estudiante debe ayudar a superar las barreras que el estudiante de educación superior encuentran en el proceso de enseñanza aprendizaje.

\section{Correspondencia:}

Rodrigo Rondón Herz

Correo electronico: drigorondon@veterinariarondon. com

\section{REFERENCIAS BIBLIOGRÁFICAS}

1. Allen, S., Blackwell, M. J., Brunk, C. G., \& Lawrence, H. (2007). Visión del Futuro de la Educación Médica Veterinaria. $J$ Vet Med Educ, 34(1), 45

2. Álvarez-Hernández, G., \& Delgado-De la Mora, J. (2015). Diseño de estudios epidemiológicos. I. El estudio transversal: Tomando una fotografía de la salud y la enfermedad. Boletín Clínico Hospital Infantil del Estado de Sonora, 32(1), 26-34.

3. Andrade, R. (2020). El enfoque de competencias y su vinculación con el constructivismo en el nivel medio superior de México. Revista educarnos, 1, 141-143. https://www.researchgate.net/publication/ 350568343 E L_ENFOQUE_DE COMPETENCIAS_Y_SU_VINCULACION CON_EL_CONSTRUCTIVISMO_EN_EL NIVEL_MĒDIO_SUPERIOR_DE_MEXICO

4. Barreda, M. (2009). Evaluación de competencias genéricas en Medicina. [Tesis de Médico Veterinario]. Universidad Nacional Mayor de San Marcos.

5. Bertone, P. A. (2019). La evaluación con rúbrica ¿Una forma de enseñar, una oportunidad de aprender? Mirada desde la innovación en cirugía. UniRío Editora.

6. Cooper, M. M., Caballero, M. D., Ebert-May, D., 
Fata-Hartley, C. L., Jardeleza, S. E., Krajcik, J. S., ... \& Underwood, S. M. (2015). Challenge faculty to transform STEM learning. Science, 350(6258), 281282.

7. Flores, F., Acosta, R., Ávila, J., Díaz, C., Flores, J., Rojas, C., \& Sáez, F. (2017). Estrategias didácticas para el aprendizaje significativo en contextos universitarios. Universidad de Concepción. http://docencia.udec.cl/unidd/images/stories/ contenido/material_apoyo/ESTRATEGIAS $\% 20$ DIDACTICAS.pdf

8. García-Carpintero, E., Siles-González, J., MartínezRoche, M. E., Martínez-Miguel, E., Manso-Perea, C., González-Cervantes, S., \& García-García, E. (2019). Percepciones de los estudiantes sobre sus vivencias en las prácticas clínicas. Enfermería universitaria, 16(3), 259-268.

9. Hidalgo, B. G., Mayacela, Á. G., \& Hidalgo, I. M. (2017). Estrategias didácticas para potenciar el aprendizaje de Farmacología clínica. Revista Habanera de Ciencias Médicas, 16(3), 439-453.

10. Izaga, W.I. (2018). Diagnóstico del desempeño académico en el área de prácticas pre profesionales de los estudiantes de medicina veterinaria en Trujillo, 2017. [Tesis Maestría]. Universidad Católica Los Ángeles de Chimbote.

11. King, A. (1993). From sage on the stage to guide on the side. College teaching, 41(1), 30-35.

12. Kim, A. M., Speed, C. J., \& Macaulay, J. O. (2019). Barriers and strategies: implementing active learning in biomedical science lectures. Biochemistry and Molecular Biology Education, 47(1), 29-40.

13. Lemos, H. M., \& Londoño, A. N. H. (2006). Construcción y validación del cuestionario de dependencia emocional en población colombiana. Acta colombiana de psicología, 9(2), 127-140.

14. López, G. E. (2016). En torno al concepto de competencia: un análisis de fuentes. Revista de Currículum y Formación de Profesorado, 20(1), 311322.

15. Mazur, E. (2009). Education: Farewell, lecture? Science, 323, 50-51.

16. Piaget, J. (1964). Part I: Cognitive development in children: Piaget development and learning. J Res Sci Teach, 2, 176-186.

17. San Martín, H. F. (2003). Homologación de planes de estudio de la carrera de medicina veterinaria en Latinoamérica. Revista de Investigaciones Veterinarias del Perú, 14 (2), 178-180.

18. Taylor, P. J. (2012). Competencias profesionales en medicina veterinaria. Asociacion Panamericana de Ciencias Veterinarias.

19. Tebar, L. (2003). El perfil del profesor mediador. Editorial Santillana.

20. Villa, A., \& Poblete, M. (2004). Practicum y evaluación de competencias. Profesorado: Revista de Currículum y Formación del Profesorado, 8 (2), $1-19$. 